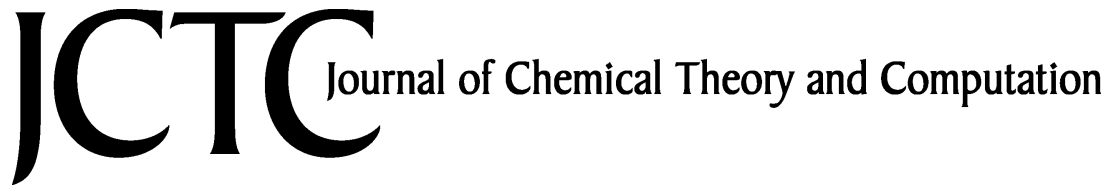

\section{Simulation of Interaction Forces between Nanoparticles: End-Grafted Polymer Modifiers}

\author{
Krishna Tej Marla and J. Carson Meredith* \\ School of Chemical and Biomolecular Engineering, Georgia Institute of Technology, \\ Atlanta, Georgia 30332-0100
}

Received May 9, 2006

\begin{abstract}
The interaction forces between nanoscale colloidal particles coated with end-grafted Lennard-Jones homopolymers are calculated using off-lattice Monte Carlo simulations in the NVT ensemble. The focus of this work is on grafted polymers that are of approximately the same size as the nanoparticle, a regime intermediate to the star-polymer and Derjaguin limits. The effects of chain length $(N)$, nanoparticle diameter $\left(\sigma_{c}\right)$, grafting density $\left(\rho_{a}\right)$, and colloidpolymer and polymer-polymer interaction energies $\left(\epsilon_{\mathrm{cp}}\right.$ and $\left.\epsilon_{\mathrm{pp}}\right)$ on the polymer-induced force between the nanoparticles are explored. The inclusion of attractive dispersion interactions between the particle and polymeric modifier results in either long-ranged attraction and shortranged repulsion or pure repulsion, depending on the molecular parameters. The polymerinduced attraction occurs even under good solvent conditions below a threshold grafting density $\left(\rho_{a}\right)$ and chain length $(N)$ and could be attributed to both bridging (colloid-polymer) and intersegmental (polymer-polymer) attraction. Above the threshold $\rho_{a}$ and $N$ values, chain entropy and excluded volume effects begin to dominate and lead eventually to polymer-induced repulsion and, consequently, nanoparticle stabilization. These results point to the importance of considering dispersion attractions between grafted segments and the nanoparticle surface in modeling these high-curvature colloid interactions.
\end{abstract}

\section{Introduction}

We previously used Monte Carlo simulation to examine homopolymer adsorption on nanoscale colloidal particles and the associated polymer-induced forces between them. ${ }^{1}$ However, polymers irreversibly end-grafted to the surface of nanoparticles are technologically more significant for passivating, stabilizing, or directing the assembly of nanoparticle dispersions. These systems have important applications in areas such as photonics and electronics, chemical and biological sensing, and energy storage. In addition, organically modified nanoparticles also serve as functionalized building blocks for programmed assembly into supramolecular entities. Models of the effect of molecular parameters (polymer chain length, graft density, nanoparticle diameter, and polymer-particle interaction energy) on the resulting interaction forces are critical for the rational design of modifiers for these purposes.

\footnotetext{
* Corresponding author e-mail: carson.meredith@ chbe.gatech.edu.
}

Planar Surface Models. The structure and physical properties of end-grafted polymers and the interactions between polymer-grafted surfaces have been studied extensively in recent years. Models of nonadsorbing end-grafted polymers (no segment-surface attraction beyond the tethered segment) predict the well-known mushroom-to-brush transition as the graft density increases. When two such polymer brushes approach in a good solvent, unfavorable steric overlaps between the polymer segments give rise to an entropic repulsion, commonly called "polymeric stabilization". These phenomena have been described successfully via scaling analysis, ${ }^{2,3}$ self-consistent field (SCF) theory, ${ }^{4-9}$ and Monte Carlo ${ }^{10,11}$ and molecular dynamics simulations. ${ }^{12,13}$ In addition, many experimental studies of the interactions between planar polymer brushes ${ }^{14-19}$ report a monotonically increasing repulsive force, concurrent with models that neglect polymer-particle attractive interactions.

Alternately, attractive interactions between the surface and the tethered polymer segments result in a more complex 
situation. At low grafting densities, the polymers adopt a flat, pancakelike conformation to maximize the favorable surface-segment contacts. As the grafting density is increased, the polymer chains form brushes, but at a relatively higher grafting density compared to the nonadsorbing surfaces. Attractive interactions between the grafted polymer and the surface can result in bridging attraction between the polymer-coated surfaces. In a recent study, the force between a spherical polymer brush in a good solvent and a coated atomic force microscope (AFM) tip indicated bridging attraction even at relatively high grafting density. ${ }^{20}$ One important question is whether the curvature of the brush influences the importance of segment-surface attractions.

Curvature Effects. SCF and scaling methods have also been extended to curved surfaces, mostly in either the starpolymer limit, where the polymers are very long compared to the particle diameter, ${ }^{21-24}$ or the Derjaguin limit, where the range of the interaction is small compared to the particle radius (small curvature effects). Nanoscale colloidal particles are often tethered to oligomers (short alkyl chains) of approximately the same size range as the nanoparticle. This regime, intermediate to the star-polymer and the Derjaguin limits, has been relatively unexplored, particularly with regard to effects of polymer-surface attraction. The primary effect of curvature, relative to flat surfaces, is that the volume available to the grafted chains increases as $\sim r^{3}$ rather than $\sim r(r=$ radial distance from the surface). As a result, entropy loss from overlapping chains is not as much of a penalty when curved brushes approach one another. The diminished role of overlap entropy points to the possibility that polymer-surface attraction might become significant, which would lead to differences in the interactions between highly curved brushes (nanoparticles) versus low-curvature or flat brushes (conventional colloid sizes $\sim 100 \mathrm{~nm}$ to $10 \mu \mathrm{m}$ ).

Wijmans et al. ${ }^{25}$ used a two-dimensional lattice SCF to calculate the interaction between two polymer-coated particles whose radii of curvature are of the same order of magnitude as the polymer layer thickness. They found the repulsive interactions to be far less than those predicted by the Derjaguin approximation. Recent Monte Carlo simulations in this regime ${ }^{26}$ predicted a lower repulsive force at short distances than the Wijmans et al. results, ${ }^{27}$ attributed to the neglect of monomer correlations in the SCF theory. The interaction forces obtained from the simulations were also modeled by a combination of the Witten-Pincus approach $^{26}$ and the Flory theory for dilute polymer solutions. $^{22}$ SCF studies of grafted-polymer nanoparticles in a good solvent ${ }^{28}$ show that the interaction can become attractive when the segment "clouds" of the two spheres just begin to overlap and can become purely attractive at low grafting densities. The reason for these contrasting experimental and modeling results is not apparent and reflects the need for further study.

In this paper, we consider the effect of end-grafted polymeric modifiers on the interaction forces between nanoparticles that (1) experience Lennard-Jones (LJ) attraction with the end-grafted polymer segments and (2) are of the same dimensions as the polymers; for example, curvature effects are important. Using Monte Carlo simulations, we

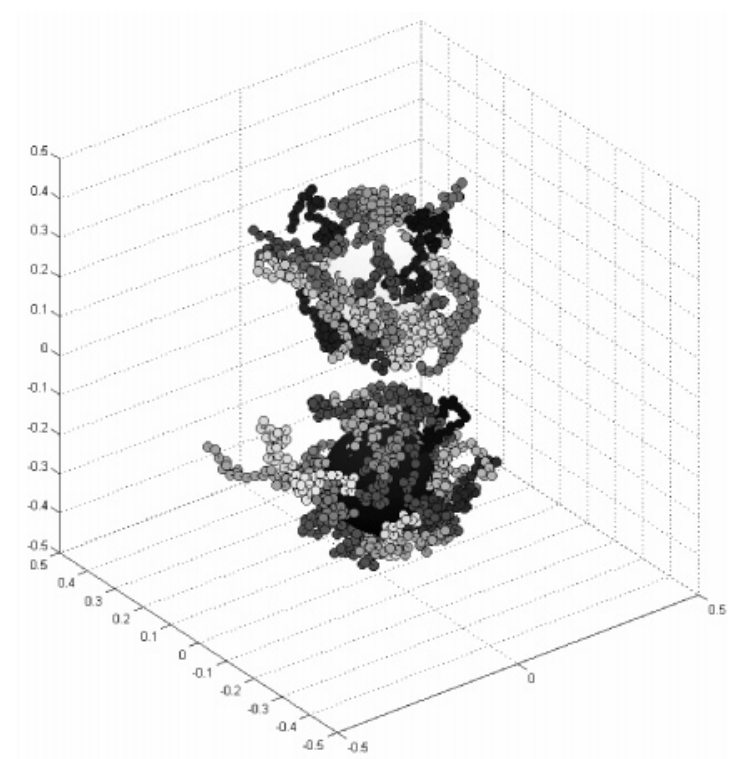

Figure 1. Configuration snapshot of the simulation box showing two colloidal nanoparticles with end-grafted polymeric modifiers $\left(\sigma_{\mathrm{c}}=10 \sigma_{\mathrm{p}}, N=30, \rho_{a}{ }^{*}=3.0, T^{\star}=3.0\right)$.

study the effect of nanoparticle diameter $\left(\sigma_{\mathrm{c}}\right)$, polymer chain length $(N)$, grafting density $\left(\rho_{a}\right)$, and colloid-polymer and polymer-polymer interaction energies $\left(\epsilon_{\mathrm{cp}}\right.$ and $\left.\epsilon_{\mathrm{pp}}\right)$ on the polymer-induced force profiles between nanoparticles. We identify the physical mechanisms that lead to attraction or repulsion in the force profiles on the basis of interplay between the various molecular parameters described above.

\section{Simulation Method}

Parameters and Model. We have simulated the interaction between two spherical nanoparticles grafted with LJ polymer chains by using three-dimensional off-lattice Monte Carlo simulations in the canonical (NVT) ensemble. Figure 1 contains a schematic of the simulation box with two nanoscale colloidal particles that are fixed along the $z$ axis. Each of the nanoparticles has $n_{\mathrm{c}}$ chains of length $N$ that are grafted at random positions on the nanoparticle surface. In most cases, $N=10-30$, while a few results are also presented for longer chains of $N=50$. To study the effect of grafting density, the number of grafting chains is varied from $n_{\mathrm{c}}=6$ to $n_{\mathrm{c}}=55$, which correspond to grafting densities ranging from 0.02 to 0.18 . The grafting density $\left(\rho_{a}\right)$ is given by $\rho_{a}=n_{\mathrm{c}} / \pi \sigma_{\mathrm{c}}{ }^{2}$. Grafting density is normalized by dividing by the bulk radius of gyration, $R_{\mathrm{g}}$, with $\rho_{a}{ }^{*}=$ $\rho_{a} \pi R_{\mathrm{g}}{ }^{2}$. A "mushroom" (or "pancake", depending on the polymer-particle interactions) to "brush" transition occurs at a reduced grafting density $\rho_{a} * \approx 1$, where chains grafted to a single surface begin to overlap laterally. The grafting densities studied in this work correspond to $\rho_{a}{ }^{*}$ values of $0.5 \leq \rho_{a} * \leq 5$, depending on chain length and nanoparticle diameter. Experimental values of $\rho_{a}{ }^{*}$ commonly range between 1 and $20 .^{29}$

The polymers are modeled as fully flexible chains with a bond length of $\sigma_{\mathrm{p}}$. The polymer segments interact via the Lennard-Jones potential, cut and shifted to zero at $r_{\mathrm{c}}=2.5 \sigma_{\mathrm{p}}$, given in eq 1 
$U\left(r_{i j}\right)=\left\{\begin{array}{r}4 \epsilon_{i j}\left[\left(\frac{\sigma_{i j}}{r_{i j}}\right)^{12}-\left(\frac{\sigma_{i j}}{r_{i j}}\right)^{6}-\left(\frac{\sigma_{i j}}{r_{c}}\right)^{12}+\left(\frac{\sigma_{i j}}{r_{c}}\right)^{6}\right] \begin{array}{r}r \leq r_{c} \\ r>r_{c}\end{array}\end{array}\right.$

where $\sigma_{i j}=\left(\sigma_{i}+\sigma_{j}\right) / 2$ is the size parameter and $\epsilon_{i j}$ is the $\mathrm{LJ}$ interaction energy parameter. The cut-and-shifted LJ potential, for which phase behavior and structure have been characterized well, is used as a computational simplification for evaluating the polymer segment-segment interactions. However, the colloid-colloid and the colloid-polymer interactions are modeled using the "full" LJ potential; for example, the potential was evaluated at all interparticle distances without a cutting off the potential. This was done to avoid computational artifacts and difficulty in interpreting results because the cutoff value, $r_{\mathrm{c}}$, would vary widely as the colloid size is varied. The interaction between the colloid and the first polymer segment that is grafted permanently to the colloid surface is not considered, as it would contribute only a constant to the energy and cancel in calculations of energy change. The temperature was reduced with the LJ parameters in the usual manner, $T^{*}=T k_{\mathrm{B}} / \epsilon_{\mathrm{pp}}$, where $\epsilon_{\mathrm{pp}}$ is the polymer segment-segment interaction parameter and $k_{\mathrm{B}}$ is the Boltzmann constant. All of the simulations were performed at $T^{*}=3.0$ (good solvent conditions), $\epsilon_{\mathrm{cc}}=1$, and $\epsilon_{\mathrm{pp}}=1$, unless specified otherwise. The polymer-coated nanoparticle interaction forces were calculated for two different particle diameters, $\sigma_{\mathrm{c}}=10 \sigma_{\mathrm{p}}$ and $5 \sigma_{\mathrm{p}}$. The effect of the colloid-polymer interaction strength $\left(\epsilon_{\mathrm{cp}}\right)$ on the force profiles was studied by varying $\epsilon_{\mathrm{cp}}$ from $\epsilon_{\mathrm{cp}}=2 \epsilon_{\mathrm{pp}}$ to a purely soft-repulsive interaction between the colloid and polymer segments. The dimensions of the simulation box are $50 \sigma_{\mathrm{p}}$ in the $x$ and $y$ directions and $60 \sigma_{\mathrm{p}}$ in the $z$ direction, large enough to prevent artificial interactions between periodic images.

It is important to understand the relevance of the chosen model to realistic experimental systems. We have not accounted for atomic or electronic detail because this work does not aim to account for the effects of chemistry or chemical differences between polymers and surfaces. Rather, the focus of this work is physical effects of curved particles that interact attractively with short polymer chains. The freely jointed LJ model is a coarse-grained approach chosen for its simplicity, its ability to capture physical behavior, and the large amount of information available for LJ model systems. The LJ potential is one of the most studied attractive potentials and has been used for decades to examine the physical behavior of pure fluids, mixtures of small molecules, polymer solutions, melts, and blends, as well as colloidal systems. The equations of state properties of LJ fluids and polymers are well-known, allowing us to accurately set the solvent quality, temperature, grafting densities, and other parameters. It is likely that our results would be most appropriately compared to experiments where oligomers are irreversibly grafted to random sites on colloidal nanoparticles, in which there is a net attraction between the chain segments and the particle. In particular, systems in which isotropic dispersion (London) forces dominate would be most similar to the model, for example, polystyrene grafted to silica in a toluene solvent. The use of freely jointed chain segments is an extreme simplification that artificially increases the number of available chain conformations (entropy) relative to more realistic bead-spring models. ${ }^{30}$ However, the inclusion of restrictions to segmental rotation tends to result in quantitative corrections to calculated properties but often does not change the underlying physical behavior.

Computational Details. The simulation was initialized by fixing the two nanoparticles at a specified distance from each other along the $z$ axis. Grafting sites were then chosen at random on each surface, and the first monomer of each polymer chain was fixed permanently at those sites. The remaining $N-1$ segments of each chain were then grown in a stretched-out conformation avoiding overlaps with neighboring chains. The initially stretched conformation facilitates faster equilibrium and helps avoid "locked" configurations. Equilibration of the grafted chains is achieved by two methods for generating chain conformations. Chain segments 2 through $N / 2$ were moved by using the "crankshaft" method, which performs a random rotation about the axis passing through the center of the neighboring segments. The remaining part of the chain farthest from the particle surface, $N / 2+1$ to $N$, was moved using the continuum configurational bias (CCB) algorithm. The CCB method consists of cutting a chain at a randomly selected site and regrowing it site by site until the original chain length is restored. The regrowth process involves scouting various trial positions on a spherical surface centered at the previous existing site, favoring nonoverlapping low-energy configurations at each stage. Details on the computer implementation of the CCB method and its applicability and limitations have been outlined in the literature. ${ }^{31,32}$ After equilibrium is achieved, the forces on each particle $\left[F_{\mathrm{AP}}(r)\right.$ and $\left.F_{\mathrm{BP}}(r)\right]$ due to the polymer chains were calculated. Such individual simulations were performed from the nearest center-to-center separation $\left(r_{\mathrm{c}-\mathrm{c}}\right)$ between the nanoparticles of $r_{\mathrm{c}-\mathrm{c}}=\sigma_{\mathrm{c}}+$ $2 \sigma_{\mathrm{p}}$ in increments of $1 \sigma_{\mathrm{p}}$ up until approximately $r_{\mathrm{c}-\mathrm{c}}=2.5$ $\sigma_{\mathrm{c}}$. The method and equations used for the force calculation are the same as those described in part I of this series. The force profiles reported in this work are normalized by subtracting the force at "infinite" separation, where the nanoparticle surfaces are far enough not to affect one another significantly.

The reaction-coordinate approach used here, where particles are constrained to approach one another along a collision path, is a common geometry in free energy and force calculations, for example, free energy perturbation and thermodynamic integration approaches. The approach has been shown to be accurate for calculating equilibrium properties of reacting species, fluid structure, and forces between submerged colloidal particles. ${ }^{33}$ The potential disadvantages include the fact that experimental systems may not really achieve equilibrium chain conformations as particles approach and collide, although in general, the time scale for chain rearrangement ( $\sim$ nanoseconds) is usually several orders of magnitude faster than the particle-particle collision time scale ( $\sim$ microseconds). The other possibility is that of frozen configurations trapped in a local energy minimum as the particles approach closely. We have 
Table 1. Simulation Conditions Explored in This Studya

\begin{tabular}{cccccccccc}
\hline $\begin{array}{c}\text { case } \\
\text { number }\end{array}$ & $\sigma_{\mathrm{c}}$ & $N$ & $\epsilon_{\mathrm{cp}}$ & $\epsilon_{\mathrm{pp}}$ & $\begin{array}{c}\rho_{\mathrm{a}}{ }^{*}= \\
\rho_{\mathrm{a}} \pi R_{\mathrm{g}}{ }^{2}\end{array}$ & $\begin{array}{c}\rho_{\mathrm{a}}= \\
n_{d} \pi \sigma_{\mathrm{c}}{ }^{2}\end{array}$ & $n_{\mathrm{c}}$ & $R_{\mathrm{g}}{ }^{2}$ & $R_{\mathrm{EED}}{ }^{2}$ \\
\hline 1 & 10 & 30 & 1.0 & 1.0 & 0.5 & 0.02 & 6 & 8.05 & 45.23 \\
2 & & & & & 1.0 & 0.04 & 11 & 8.15 & 47.33 \\
3 & & & & & 2.0 & 0.07 & 22 & 8.16 & 48.16 \\
4 & & & & & 3.0 & 0.11 & 33 & 8.33 & 50.63 \\
5 & & & & & 5.0 & 0.18 & 55 & 8.86 & 57.90 \\
6 & 10 & 30 & 2.0 & 1.0 & 5.0 & 0.18 & 55 & 8.16 & 48.91 \\
7 & & & Rep & 1.0 & 5.0 & 0.18 & 55 & 9.95 & 72.11 \\
8 & & & 1.0 & Rep & 5.0 & 0.18 & 55 & 15.82 & 127.71 \\
9 & & & Rep & Rep & 5.0 & 0.18 & 55 & 16.44 & 137.02 \\
10 & 10 & 10 & 1.0 & 1.0 & 0.8 & 0.11 & 33 & 2.42 & 15.12 \\
11 & 10 & 50 & 1.0 & 1.0 & 5.0 & 0.11 & 33 & 14.74 & 86.19 \\
12 & 5 & 10 & 1.0 & 1.0 & 0.8 & 0.11 & 8 & 2.42 & 14.94 \\
13 & 5 & 30 & 1.0 & 1.0 & 3.0 & 0.11 & 8 & 8.54 & 51.55 \\
\hline
\end{tabular}

${ }^{a} \rho_{a}\left(=n_{c} / \pi \sigma_{c}{ }^{2}\right)$ is the grafting density, and $\rho_{a}{ }^{*}\left(=\rho_{a} \pi R_{g}{ }^{2}\right)$ is the reduced grafting density. $n_{\mathrm{c}}$ is the number of chains grafted on each colloidal particle. $R_{\mathrm{g}}$ and $R_{\mathrm{EED}}$ are the radius of gyration and end-toend distance of the end-grafted polymer chains. ${ }^{b}$ "Rep" indicates a purely repulsive interaction obtained by using only the repulsive term in the Lennard-Jones 6-12 potential.

attempted to circumvent this by running each simulation multiple times from different initial configurations.

Equilibration required $(20-40) \times 10^{6}$ Monte Carlo steps (where each step corresponds to one attempted move) depending on the grafting density and chain length. "Equilibrium" was defined as occurring when the energy approached an asymptote and fluctuated less than $2 \%$ and did so in a repeatable manner from different initial states. Average forces, chain conformational properties, and segment density profiles were collected over $(100-160) \times 10^{6}$ attempted moves at each separation distance. The statistical error for the conformational properties of the chains was taken as the root-mean-square fluctuation divided by the square root of the number of independent blocks. Statistical error in the forces was based on the statistical inefficiency parameter discussed by Allen and Tildesley. ${ }^{34}$ Error bars were omitted from the figures if they were smaller than the symbols representing the data points. Table 1 gives the details of all of the cases that were investigated in this study.

\section{Results and Discussion}

Effect of Grafting Density $\left(\rho_{a}^{*}\right)$. Figure 2 shows the effect of reduced grafting density $\left(\rho_{a}^{*}\right)$ on the polymer-induced forces $\left(F_{\mathrm{P}}\right)$ between the nanoparticles at $\sigma_{\mathrm{c}}=10 \sigma_{\mathrm{p}}, N=30$, $\epsilon_{\mathrm{cp}}=\epsilon_{\mathrm{pp}}=1$. The direct $\mathrm{LJ}$ dispersion force between the uncoated nanoparticles obtained by

$$
F_{D}(r)=-\frac{\partial U_{\mathrm{cc}}(r)}{\partial r}=\frac{48}{r}\left[\left(\frac{\sigma_{\mathrm{c}}}{r}\right)^{12}-0.5\left(\frac{\sigma_{\mathrm{c}}}{r}\right)^{6}\right]
$$

is also shown for comparison. In all force profiles reported in this paper, the $x$ coordinate is taken to be $D=\left(r_{\mathrm{c}-\mathrm{c}}-\right.$ $\sigma_{\mathrm{c}}$ ), which is the nearest surface-to-surface distance between the nanoparticles. For all grafting densities, the forcedistance plots show long-ranged attraction and short-ranged repulsion, similar in form and comparable in magnitude to the direct nanoparticle force. The attractive minimum grows deeper and shifts to larger separations with increasing

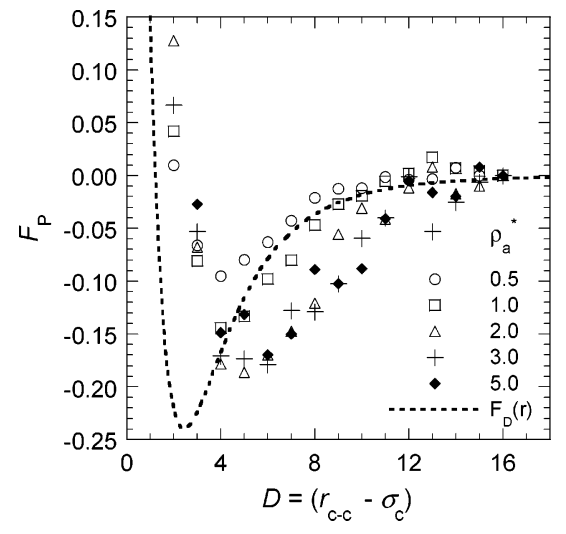

Figure 2. Force-distance plots [polymer-mediated force $F_{\mathrm{P}}(r)$ vs $D$ ] at different grafting densities ranging from $\rho_{a}{ }^{*}=0.5$ to $\rho_{\mathrm{a}}{ }^{*}=$ 5.0. $\sigma_{\mathrm{c}}=10 \sigma_{\mathrm{p}}, N=30$, and $\epsilon_{\mathrm{cp}}=\epsilon_{\mathrm{pp}}=1$. The direct force between the bare nanoparticles $\left(F_{\mathrm{D}}\right)$ calculated from eq 2 is also shown for comparison.

grafting densities up to $\rho_{a}{ }^{*}=3.0$, beyond which the minimum shifts back upward (for $\rho_{a}^{*}=5.0$ ). The nature and magnitude of the polymer-induced forces between the nanoparticles are a consequence of the competition between attraction due to bridging and intersegmental interactions and repulsion due to steric interactions between the grafted chains. Bridging occurs because of attractive segmentsurface interactions and the presence of exposed adsorption sites on the nanoparticle surface. At $\rho_{a}{ }^{*}=0.5$, the polymer is in a pancakelike conformation wherein the average distance between the grafting sites is more than the $R_{\mathrm{g}}$ of the polymer chains and the grafted polymers tend to lie flat on the particle surface because of favorable particle-polymer contacts. Although the surface coverage is low (which is favorable for bridging), the small number of grafted chains results in a very low density of bridges formed. In addition, the flattened conformations of the grafted polymers also constrain the amount of bridging that can occur. As $\rho_{a}{ }^{*}$ is increased beyond $\rho_{a}{ }^{*}=1.0$, the polymer chains undergo a gradual transition to the brush regime, wherein the excluded volume considerations cause the polymer chains to extend outward from the surface. It is noteworthy that the pancake (or mushroom) to brush transition for short chains (low molecular weight polymers) is not a very sharp transition and occurs over a broad range of grafting densities. ${ }^{35}$ With increasing $\rho_{a}{ }^{*}$, there are more chains available that can form bridges between the two particles. However, there is a progressive decrease in the availability of binding sites on the surface and also an increase in the monomer density in the interparticle region. These competing effects suggest that there is a threshold value of grafting density beyond which the number of bridging chains would be limited by entropic effects.

Figure 3 shows the average number of bridging chains $\left(n_{\text {bridge }}\right)$ versus the separation distance $(D)$ at different grafting densities corresponding to Figure 2. The plots indicate that there is a maximum in $n_{\text {bridge }}$ at a grafting density of $\rho_{a}{ }^{*}=$ 3.0, and a further increase in the grafting density results in a slight decrease in $n_{\text {bridge }}$. The grafting density at which the polymer-induced attraction between the nanoparticles is strongest coincides with the maximum in $n_{\text {bridge }}$ (at $\rho_{a}{ }^{*}=$ 


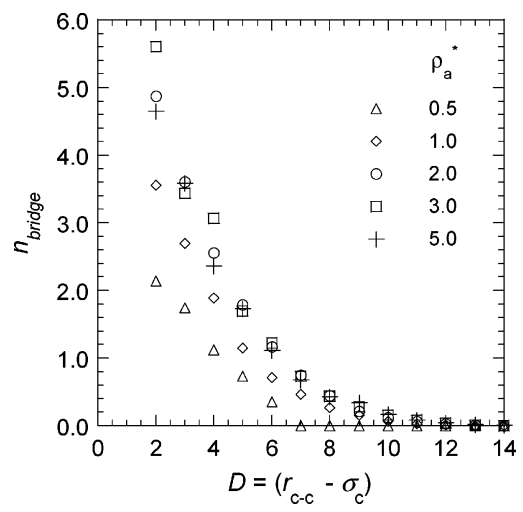

Figure 3. Number of bridging chains versus surface-tosurface separation distance $\left(D=r_{\mathrm{c}-\mathrm{c}}-\sigma_{\mathrm{c}}\right)$ at different grafting densities ranging from $\rho_{a}{ }^{*}=0.5$ to $\rho_{a}{ }^{*}=5.0 . \sigma_{\mathrm{c}}=10 \sigma_{\mathrm{p}}, N=$ 30 , and $\epsilon_{\mathrm{cp}}=\epsilon_{\mathrm{pp}}=1$.

3.0), suggesting that bridging is responsible primarily for the attraction in the force profiles. Also, at a constant grafting density, the number of bridging chains increases with decreasing interparticle separation, suggesting a monotonic attraction in the force profiles. However, at close separations, steric effects dominate the bridging attraction resulting in polymer-induced repulsion between the nanoparticles. While the intersegmental interactions between the grafted polymer chains also contribute to the polymer-induced attraction, it is difficult to quantify exactly the relative contributions of bridging and segmental interactions to the overall attraction. The effect of polymer segment-segment interactions on the polymer-induced forces is explored in greater detail later in this section. The decrease in the magnitude of the attractive minimum as $\rho_{a}{ }^{*}$ is increased from $\rho_{a}{ }^{*}=3.0$ to $\rho_{a}{ }^{*}=5.0$ suggests that further increasing the grafting density may eventually result in the repulsive entropic effects dominating the attraction leading to monotonic repulsion in the force profiles. The separation distance between the nanoparticles at which $F_{\mathrm{P}}$ changes sign (i.e., $F_{\mathrm{P}}=0$ ) and the location of the attractive minimum are related closely to the conformations of the end-grafted polymers. The mean-squared endto-end distance $\left(R_{\mathrm{EED}}{ }^{2}\right)$ and the radius of gyration squared $\left(R_{\mathrm{g}}{ }^{2}\right)$ of the polymer chains at different grafting densities are shown in Table 1 . The reported values of $R_{\mathrm{EED}}{ }^{2}$ and $R_{\mathrm{g}}{ }^{2}$ are obtained by averaging over all nanoparticle separation distances at each grafting density. The bulk values of $R_{\mathrm{EED}}{ }^{2}$ and $R_{\mathrm{g}}{ }^{2}$ (for $N=30$ ) are 53.2 and 8.96, respectively. For grafting densities up to $\rho_{a}^{*}=3.0$ (at $\epsilon_{\mathrm{cp}}=\epsilon_{\mathrm{pp}}=1$ ), $R_{\mathrm{EED}}{ }^{2}$ and $R_{\mathrm{g}}{ }^{2}$ are less than the bulk values due to the attractive interactions between the nanoparticle and the grafted chains, which cause the polymers to adopt more compact conformations near the particle. However at $\rho_{a}{ }^{*}=5.0, R_{\mathrm{EED}}{ }^{2}$ is more than the bulk value, strengthening the argument that the excluded volume effects begin to dominate at this grafting density. The monotonic increase in $R_{\mathrm{EED}}{ }^{2}$ and $R_{\mathrm{g}}{ }^{2}$ with increasing $\rho_{a}{ }^{*}$ is responsible for the shift in the location of the attractive minimum and the thermodynamic minimum (location at which $F_{\mathrm{P}}=0$ ) to larger separation distances.

Effect of Particle Diameter $\left(\sigma_{c}\right)$ and Chain Length $(N)$. Figure 4 shows a plot of force due to the grafted chains $\left(F_{\mathrm{P}}\right)$ between two nanoparticles for diameters of $\sigma_{c}=10 \sigma_{\mathrm{p}}$ (Figure
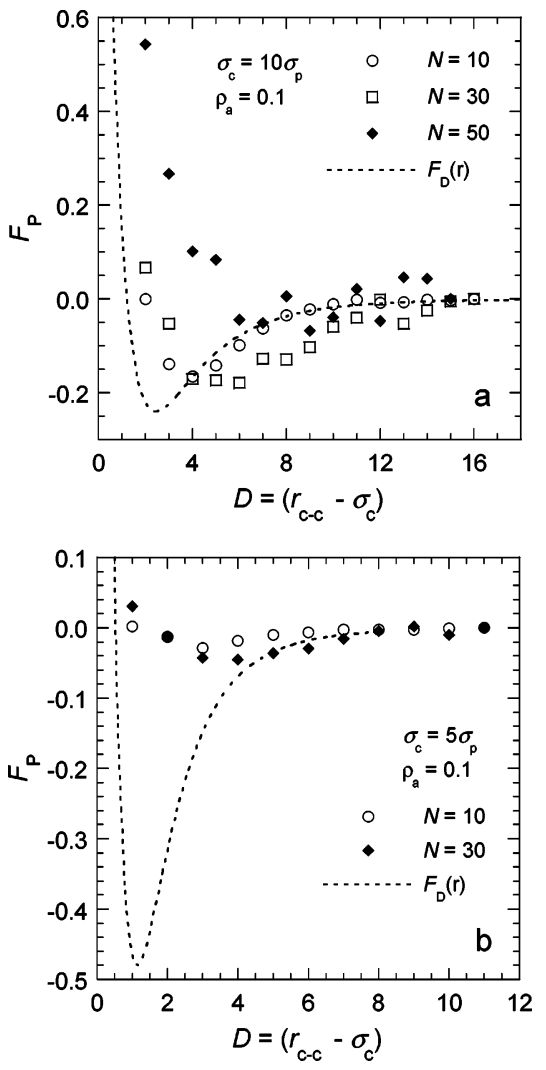

Figure 4. Force-distance plots [polymer mediated force $F_{\mathrm{P}}(r)$ vs $D$ ] for diameters of $\sigma_{\mathrm{c}}=10 \sigma_{\mathrm{p}}$ (a) and $5 \sigma_{\mathrm{p}}$ (b) at different chain lengths. $\epsilon_{\mathrm{cp}}=\epsilon_{\mathrm{pp}}=1$. The grafting density is constant at $\rho_{a}=0.1$ for all cases. Note however that $\rho_{a}{ }^{*}$ varies with $\sigma_{\mathrm{c}}$ and $N$ (see Table 1). The direct force between the bare nanoparticles $\left(F_{\mathrm{D}}\right)$ calculated from eq 2 is also shown for comparison.

$4 \mathrm{a})$ and $5 \sigma_{\mathrm{p}}$ (Figure $4 \mathrm{~b}$ ) at different chain lengths and $\epsilon_{\mathrm{cp}}=$ $\epsilon_{\mathrm{pp}}=1$. The effect of chain length was studied by keeping the number of grafted chains per particle constant at all chain lengths. This implies that, although the grafting density $\rho_{a}$ $\left(=n_{\mathrm{c}} / \pi \sigma_{\mathrm{c}}^{2}\right)$ remained constant at each particle size $\left(\rho_{a}=\right.$ $0.1)$, the reduced grafting density $\rho_{a}{ }^{*}\left(=\rho_{a} \pi R_{\mathrm{g}}{ }^{2}\right)$ varied depending on the chain length. At $\sigma_{\mathrm{c}}=10 \sigma_{\mathrm{p}}$, the attractive minimum becomes deeper and shifts to a larger distance as $N$ increases from $N=10$ to $N=30$. Further increasing the chain length to $N=50$ results in a polymer-induced force that oscillates between attraction and repulsion for $D>6 \sigma_{\mathrm{p}}$ and that is purely repulsive for $D<6 \sigma_{\mathrm{p}}$. In addition, the magnitude of the attractive minimum in $F_{\mathrm{P}}$ for $N=50$ is less compared to that for $N=30$. Figure 5 shows $n_{\text {bridge }}$ versus the separation distance for all of the $N$ and $\sigma_{\mathrm{c}}$ values corresponding to the force-distance curves in Figure 4. For $\sigma_{\mathrm{c}}=10 \sigma_{\mathrm{p}}, n_{\text {bridge }}$ is higher for longer chains, suggesting that attraction due to bridging increases monotonically with chain length. However, as chain length is increased at constant $\rho_{a}$, there is a larger number of polymer segments in the region between the two particles, resulting in increased excluded volume effects. The force plots suggest that, at $N=50$, these entropic effects tend to dominate the increased attraction due to bridging and intersegmental interactions, resulting in relatively long-range repulsion and a higher attractive minimum. At $\sigma_{\mathrm{c}}=5 \sigma_{\mathrm{p}}$, the force due to the polymer is very 


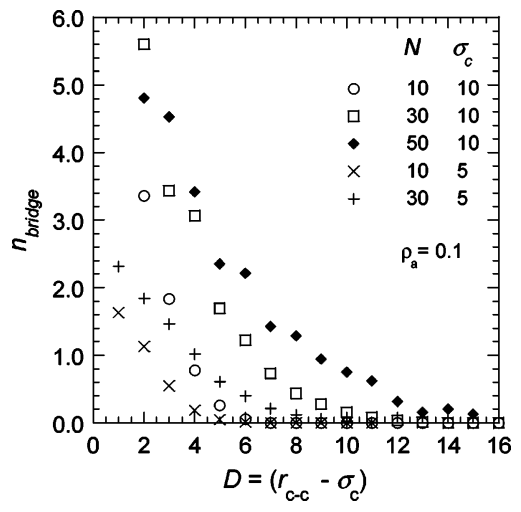

Figure 5. Number of bridging chains versus surface-tosurface separation distance $\left(D=r_{\mathrm{c}-\mathrm{c}}-\sigma_{\mathrm{c}}\right)$ at different $\sigma_{c}$ and $N$ values corresponding to the force profiles in Figure 4 . The grafting density is constant at $\rho_{a}=0.1$ and $\epsilon_{\mathrm{cp}}=\epsilon_{\mathrm{pp}}=1$.

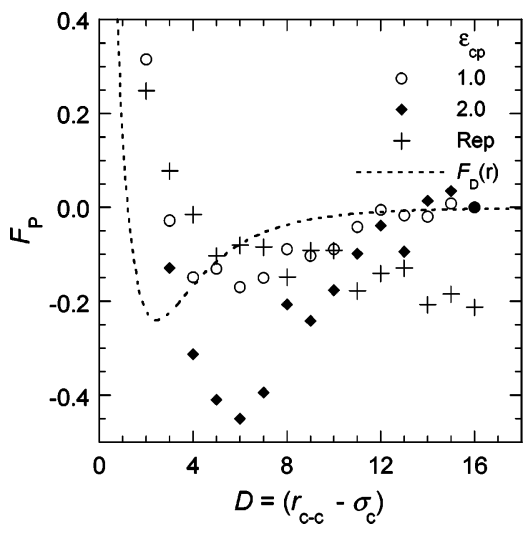

Figure 6. Force-distance plots [polymer-mediated force $F_{\mathrm{P}}(r)$ vs $D$ ] for different values of the colloid-polymer interaction strength $\left(\epsilon_{\mathrm{cp}}\right) . \sigma_{\mathrm{c}}=10 \sigma_{\mathrm{p}}, N=30, \rho_{\mathrm{a}}{ }^{*}=5.0$, and $\epsilon_{\mathrm{pp}}=1$ for all cases. "Rep" stands for the purely repulsive interaction obtained by using only the repulsive term in the LennardJones 6-12 potential. The direct force between the bare nanoparticles $\left(F_{\mathrm{D}}\right)$ calculated from eq 2 is also shown for comparison.

small compared to the direct nanoparticle force for both chain lengths $(N=10$ and $N=30)$. For a constant grafting density $\left(\rho_{a}=0.1\right)$, the number of grafting chains decreases with decreasing particle size, and hence, at $\sigma_{\mathrm{c}}=5 \sigma_{\mathrm{p}}$, the polymerinduced attraction is weaker compared to that at $\sigma_{\mathrm{c}}=10 \sigma_{\mathrm{p}}$. In addition, the decrease in the range of the $\mathrm{LJ}$ potential energy with decreasing $\sigma_{\mathrm{c}}$ results in a lesser number of bridging chains (shown in Figure 5) and consequently a weaker polymer-induced attraction.

Effect of Colloid-Polymer Interaction Parameter $\left(\epsilon_{\mathrm{cp}}\right)$. Figure 6 explores the effect of tuning the strength of the colloid-polymer interaction on the force-distance plots at $\sigma_{\mathrm{c}}=10 \sigma_{\mathrm{p}}, N=30, \epsilon_{\mathrm{pp}}=1$, and $\rho_{a} *=5.0$. To ensure that the polymers are in the brush regime, we use the highest grafting density explored in our simulations $\left(\rho_{a} *=5.0\right)$. In addition, we also ensured that the initial grafting sites were the same for all of the cases studied here. As $\epsilon_{\mathrm{cp}}$ is increased from $\epsilon_{\mathrm{cp}}=1 \epsilon_{\mathrm{pp}}$ to $\epsilon_{\mathrm{cp}}=2 \epsilon_{\mathrm{pp}}$, the attractive minimum grows deeper because of an increase in the number of bridging chains. The increased colloid-polymer interaction strength causes an increase in $n_{\text {bridge}}$, shown in Figure 7 . Also, $R_{\mathrm{EED}}{ }^{2}$

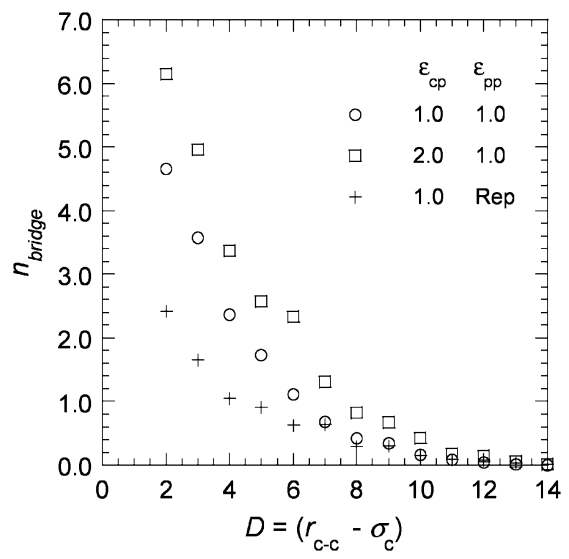

Figure 7. Number of bridging chains versus surface-tosurface separation distance $\left(D=r_{\mathrm{c}-\mathrm{c}}-\sigma_{\mathrm{c}}\right)$ at $\sigma_{\mathrm{c}}=10 \sigma_{\mathrm{p}}, N=$ 30 , and different values of $\epsilon_{\mathrm{cp}}$ and $\epsilon_{\mathrm{pp}}$. The grafting density is constant at $\rho_{a}{ }^{*}=5.0$. "Rep" stands for the purely repulsive interaction obtained by using only the repulsive term in the Lennard-Jones 6-12 potential.

and $R_{\mathrm{g}}{ }^{2}$ decrease (brush contracts) with increasing $\epsilon_{\mathrm{cp}}$, which results in the transition from attractive to repulsive force occurring at shorter separations. There is no significant difference, however, in the location of the attractive minimum.

We also studied the effect of turning off the colloidpolymer attraction by using only the repulsive part of the LJ 6-12 potential $\left(\epsilon_{\mathrm{cp}}=\right.$ "rep") to model the colloidpolymer interactions. This allows us to eliminate attraction due to bridging and isolate the effect of polymer intersegmental attractions on the force profiles. Figure 6 shows that the force due to the polymers oscillates slightly (with a periodicity of $\approx 3 \sigma_{\mathrm{p}}$ ) but grows increasingly repulsive as the particles approach each other. It is noteworthy that, for all other cases explored in this paper (wherein $F_{\mathrm{P}}$ is attractive at some distance), the force due to the polymers passes through a minimum. The occurrence of negative force at a large distance (for $\epsilon_{\mathrm{cp}}=$ "rep") is due to the repulsive colloid-polymer interactions, which drives the grafted chains to extend out as far as possible from the particle surface. Note that the $R_{\mathrm{EED}}{ }^{2}$ and $R_{\mathrm{g}}{ }^{2}$ values for this case are larger than those for any of the attractive $\epsilon_{\mathrm{cp}}$ cases (Table 1). In addition, no bridging attraction was observed for this case ( $\left.n_{\text {bridge }}=0\right)$. The only contribution to the negative force is from the polymer intersegmental attraction. (It must be noted that the repulsion between the colloid and the polymer segments enhances the range and magnitude of the intersegmental attractions compared to the case when $\epsilon_{\mathrm{cp}}=$ $\epsilon_{\mathrm{pp}}=1$.) However, at $\epsilon_{\mathrm{cp}}=\epsilon_{\mathrm{pp}}$, the polymer segments experience favorable attractive interactions with both the colloid and each other. This reduces the relative contribution of intersegmental attractions to the overall attraction.

Effect of Polymer-Polymer Interaction Parameter $\left(\epsilon_{\mathrm{pp}}\right)$. Figure 8 shows the force profiles at $\epsilon_{\mathrm{pp}}=1$ and $\epsilon_{\mathrm{pp}}=$ "rep" (soft-repulsive polymer segment interactions). The colloid-polymer interaction strength is kept constant at $\epsilon_{\mathrm{cp}}=1$ for both cases. When the polymer-polymer interactions are purely repulsive, the magnitude of polymerinduced attraction is lower compared to that in the case of 


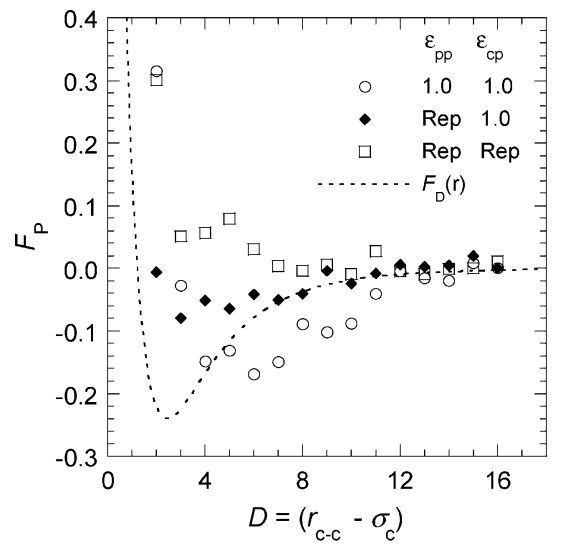

Figure 8. Force-distance plots [polymer mediated force $F_{\mathrm{p}}(r)$ vs $D$ ] for different values of the polymer-polymer interaction strength $\left(\epsilon_{\mathrm{pp}}\right) . \sigma_{\mathrm{c}}=10 \sigma_{\mathrm{p}}, N=30$, and $\rho_{\mathrm{a}}{ }^{*}=5.0$ for all cases. "Rep" stands for the purely repulsive interaction obtained by using only the repulsive term in the Lennard-Jones 6-12 potential. The direct force between the bare nanoparticles $\left(F_{\mathrm{D}}\right)$ calculated from eq 2 is also shown for comparison.

$\epsilon_{\mathrm{cp}}=\epsilon_{\mathrm{pp}}=1$ because there is no longer any contribution of intersegmental attractions to the overall force profile. The attraction that is observed in this case is solely due to bridging (because $\epsilon_{\mathrm{cp}}=1$ ). However, the bridging attraction observed here is less than that in the case of $\epsilon_{\mathrm{cp}}=\epsilon_{\mathrm{pp}}=1$ (see Figure 7) because the polymer-polymer repulsion limits the number of polymer chains that interpenetrate the opposite grafted layer. These results suggest that there is a complex interplay of the effects of $\epsilon_{\mathrm{cp}}$ and $\epsilon_{\mathrm{pp}}$, and both these parameters need to be accounted for explicitly in order to accurately model organically modified nanoparticle systems. We also calculated the force profiles for the limiting case of repulsive interactions between both colloid-polymer and polymer-polymer interactions. This represents the condition of athermal polymer chains grafted to a hard colloid surface. As expected, the polymer-induced forces are purely repulsive, in agreement with previous simulation results of interactions between spherical brushes modeled using the hard-sphere potential. ${ }^{36}$ In addition, the polymer chains are in the most stretched-out conformations as the $R_{\mathrm{EED}}{ }^{2}$ and $R_{\mathrm{g}}{ }^{2}$ values for this case are larger than those for any other condition at $\rho_{a}{ }^{*}=5.0$ (see Table 1). Also, comparing this athermal system with the case of $\epsilon_{\mathrm{cp}}=\epsilon_{\mathrm{pp}}=1$ at the same grafting density $\left(\rho_{a}^{*}=5.0\right)$ suggests that relatively higher grafting densities would be required for the steric stabilization of nanoparticles when the colloid-polymer and polymerpolymer dispersion interactions are taken into account.

Attractive forces between polymer brushes are not usually observed in experimental studies. Most previous experiments report a monotonically increasing repulsive force which decreases exponentially with increasing distance. However, bridging attraction is sometimes observed in the case of telephonic (functionalized at both ends) polymers at low surface coverage. ${ }^{27}$ These experimental studies are invariably in the limit of flat planar brushes. Recently, Goodman and co-workers ${ }^{20}$ used the AFM to measure the interactions between spherical polymer brushes and a silicon nitride tip in aqueous media. They observed a long-ranged bridging force at a low grafting density and a monotonically increasing repulsive force at higher grafting densities. In addition, the grafting density at which the bridging force was observed also varied with the polymer chemistry. The authors suggest that, in addition to the polydispersity of the polymer that causes the AFM tip to infrequently sample long polymer chains, the curvature of the latex particles may also be partially responsible for the anomalous bridging attraction. While the experimental system is different from our simulations in that we calculate the interaction force between two brushes as compared to the AFM experiments which measure the force between a brush and a surface, a qualitative comparison could still be made. The effect of different polymers used in the experiments could be compared to the effect of varying the colloid-polymer interaction parameter $\left(\epsilon_{\mathrm{cp}}\right)$ in our simulations. From Figure 6, at a constant grafting density, the attractive minimum in the force profile grows deeper with increasing $\epsilon_{\mathrm{cp}}$, suggesting that a higher grafting density would be required for stabilization. A similar observation has been reported in the AFM study wherein poly $(N$-isopropyl acrylamide) exhibited a stronger affinity for the AFM tip than poly $(N, N$-dimethyl acrylamide $)$ at the same grafting density. These results underscore the importance of incorporating van der Waals dispersion interactions in models and theories for studying nanoparticle-polymer systems.

\section{Conclusions}

The force between two nanoscale colloidal particles coated with end-grafted polymeric modifiers has been calculated using continuum Monte Carlo simulations in the NVT ensemble. The inclusion of LJ dispersion interactions results in long-ranged attraction as well as short-ranged repulsion in the $F_{\mathrm{P}}$ profiles. Polymer-induced attraction was observed under good solvent (or temperature) conditions for the polymeric modifier and when there was no energetic preference for segment-segment versus segment-surface interactions, for example, $\mathrm{LJ}$ terms $\epsilon_{\mathrm{cp}}=\epsilon_{\mathrm{pp}}$. This is also before the effect of particle-particle (bare surface) attraction is considered. Apparently, the polymer-induced attraction is attributed to both bridging and intersegmental interactions, the relative contributions of which can be adjusted with $\epsilon_{\mathrm{cp}}$ and $\epsilon_{\mathrm{pp}}$. The increased volume available to chain segments at highly curved surfaces may lead to an increased significance of polymer-particle attraction. A minimum $N$ and $\rho_{a}{ }^{*}$ is required for nanoparticle stabilization even under good solvent conditions. Beyond the minimum $N$ and $\rho_{a}{ }^{*}$, excluded volume effects begin to dominate the attractive forces, leading ultimately to purely repulsive forces (and thereby stabilization of the nanoparticles).

These results contrast with what has been observed previously in grafted-chain simulations and models for flat or curved surfaces neglecting attractive interactions between the grafted polymer and the particle surface. The graftedpolymer dispersion attraction with the surface of highly curved nanoparticles is physically significant because it causes polymer-induced attraction in situations where larger, lower-curvature colloids would remain repulsive. We note that this result has been shown here only for the LJ attraction, 
which certainly is far from reality in many experimental systems. However, the results nevertheless should motivate further study of the effects of grafted modifier-particle attraction on particle stability models, and the applicability of flat-brush models to nanoparticle stabilization.

The ability of end-grafted polymers to induce both attractive and repulsive interactions between the nanoparticles makes them particularly useful for either directed-assembly or stabilization purposes. The location and depth of the attractive minimum in the force profiles can be controlled by varying the polymer chain length, grafting density, and the relative values of colloid-polymer and polymerpolymer interaction strength. In addition, these parameters also control the particle equilibrium spacing which corresponds to $F_{\mathrm{P}}=0$. Thus, by tuning the various molecular parameters $\left(\rho_{a}^{*}, N, \epsilon_{\mathrm{cp}}\right.$, and $\left.\epsilon_{\mathrm{pp}}\right)$ of end-grafted modifiers, it is perhaps possible to achieve precise control over nanoparticle alignment and spacing in thermodynamically driven assembly processes.

Freely adsorbing homopolymer modifiers studied in part I of this series also showed a similar dependence of the polymer-induced forces on particle diameter, polymer chain length, and colloid-polymer interaction strength. However, over the range of chain lengths, $\epsilon_{\mathrm{cp}}$ and $\epsilon_{\mathrm{pp}}$, that were investigated, it was found that obtaining repulsion with homopolymer modifiers was significantly more difficult. Hence, adsorbing homopolymers are not suited ideally for stabilization purposes.

\section{References}

(1) Marla, K. T.; Meredith, J. C. Langmuir 2005, 27, 487-497.

(2) Alexander, S. J. Phys. (Paris) 1977, 38, 983-987.

(3) de Gennes, P.-G. Macromolecules 1980, 13, 1069-1075.

(4) Milner, S. T.; Witten, T. A. J. Phys. (Paris) 1988, 49 (11), 1951-62.

(5) Milner, S. T.; Witten, T. A.; Cates, M. E. Macromolecules 1988, 21 (8), 2610-19.

(6) Milner, S. T.; Witten, T. A.; Cates, M. E. Europhys. Lett. 1988, 5 (5), 413-18.

(7) Milner, S. T.; Witten, T. A.; Cates, M. E. Macromolecules 1989, 22 (2), 853-61.

(8) Zhulina, E. B.; Borisov, O. V.; Priamitsyn, V. A. J. Colloid Interface Sci. 1990, 137 (2), 495-511.

(9) Wijmans, C. M.; Scheutjens, J. M. H. M.; Zhulina, E. B. Macromolecules 1992, 25, 2657-2665.

(10) Toral, R.; Chakrabarti, A.; Dickman, R. Phys. Rev. E: Stat. Phys., Plasmas, Fluids, Relat. Interdiscip. Top. 1994, 50 (1), $343-8$.

(11) Lai, P. Y.; Binder, K. J. Chem. Phys. 1991, 95 (12), 928899.

(12) Murat, M.; Grest, G. S. Phys. Rev. Lett. 1989, 63 (10), 10747.
(13) Murat, M.; Grest, G. S. Macromolecules 1989, 22 (10), 4054-9.

(14) O'Shea, S. J.; Welland, M. E.; Rayment, T. Langmuir 1993, 9, 1826-1835.

(15) Yamamoto, S.; Ejaz, M.; Tsujii, Y.; Fukuda, T. Macromolecules 2000, 33 (15), 5608-5612.

(16) Yamamoto, S.; Ejaz, M.; Tsujii, Y.; Matsumoto, M.; Fukuda, T. Macromolecules 2000, 33 (15), 5602-5607.

(17) Yamamoto, S.; Tsujii, Y.; Fukuda, T. Macromolecules 2000, 33 (16), 5995-5998.

(18) Taunton, H. J.; Toprakcioglu, C.; Fetters, L. J.; Klein, J. Nature 1988, 332 (6166), 712-714.

(19) Taunton, H. J.; Toprakcioglu, C.; Fetters, L. J.; Klein, J. Macromolecules 1990, 23, 571-580.

(20) Goodman, D.; Kizhakkedathu, J. N.; Brooks, D. E. Langmuir 2004, 20, 2333-2340.

(21) Daoud, M.; Cotton, J. P. J. Phys. (Paris) 1982, 43 (3), 5318.

(22) Witten, T. A.; Pincus, P. A. Macromolecules 1986, 19 (10), 2509-13.

(23) Toral, R.; Chakrabarti, A. Phys. Rev. E: Stat. Phys., Plasmas, Fluids, Relat. Interdiscip. Top. 1993, 47 (6), 4240-6.

(24) Lin, E. K.; Gast, A. P. Macromolecules 1996, 29 (1), 3907.

(25) Wijmans, C. M.; Leermakers, F. A. M.; Fleer, G. J. Langmuir 1994, 10 (12), 4514-16.

(26) Cerda, J. J.; Sintes, T.; Toral, R. Macromolecules 2003, 36, 1407-1413.

(27) Flory, P. J. Principles of Polymer Chemistry; Cornell University Press: London, 1953.

(28) Roan, J.-R. Phys. Rev. Lett. 2001, 86 (6), 1027-1030.

(29) Baranowski, R.; Whitmore, M. D. J. Chem. Phys. 1995, 103 (6), 2343-2353.

(30) Kreer, T.; Metzger, S.; Muller, M.; Binder, K.; Baschnagel, J. J. Chem. Phys. 2003, 120, 4012-4023.

(31) Siepmann, J. I.; Frenkel, D. Mol. Phys. 1992, 75, 59-70.

(32) de Pablo, J. J.; Laso, M.; Suter, U. W.; Cochran, H. D. Fluid Phase Equilib. 1993, 83, 323-331.

(33) Mezei, M.; Beveridge, D. L. Ann. N. Y. Acad. Sci. 1986, $482,1-21$.

(34) Allen, M. P.; Tildesley, D. J. Computer Simulation of Liquids; Clarendon Press: Oxford, U. K., 1987.

(35) Carignano, M. A.; Szleifer, I. Macromolecules 1995, 28 (9), 3197-3204.

(36) Eiser, E.; Klein, J.; Witten, T. A.; Fetters, L. J. Phys. Rev. Lett. 1999, 82, 5076-5079.

CT6001626 